\title{
Eficacia de la intervención Motivacional y la Terapia Cognitivo- conductual para el tratamiento del Juego Patológico
} The efficacy of Motivational Intervention and Cognitive-
Behavioral Therapy for Pathological Gambling

\author{
anna Garcia-Caballero*, Marina Torrens-Lluch*, Isabel Ramírez-GendraU*, Gemma Garrido**, \\ Vicenç Vallès*,**, Núria Aragay*. \\ * Unidad de Atención al Juego Patológico, Ámbito de Atención a la Salud Mental, Consorci Sanitari de Terrassa; ** Servicio de \\ Rehabilitación Comunitaria, Ámbito de Atención a la Salud Mental, Consorci Sanitari de Terrassa.
}

\section{Resumen}

El propósito del presente estudio es analizar la eficacia de una intervención psicológica individual basada en Entrevista Motivacional y Terapia cognitivo-conductual en el tratamiento del Juego Patológico, y evaluar la eficacia de esta intervención psicológica sobre la impulsividad y la calidad de vida de los pacientes. La muestra se compone de 18 pacientes atendidos en la Unidad de Juego Patológico del Consorci Sanitari Terrassa, diagnosticados de Juego Patológico, y sin comorbilidad asociada. La media de edad fue de 46 años (SD=12). Todos ellos alcanzaron la abstinencia y se encontraban en fase de seguimiento. Se administraron pre y post tratamiento los siguientes cuestionarios: Screen for Gambling Problems (NODS), Quality of Life Enjoyment and Satisfaction (Q-LES-Q), UPPS-P Impulsive Behavior Scale, Sheehan Disability Inventory (SDI), Beck Depression Inventory (BDI), Statetrait Anxiety Inventory (STAI). Los resultados muestran una reducción significativa de los problemas asociados a la conducta de juego $(\mathrm{p}<.000)$. También se encontraron diferencias significativas en la impulsividad (UPPS-P) pre-post: urgencia negativa $(\mathrm{p}<.001)$, urgencia positiva $(\mathrm{p}<.001)$, (falta de) premeditación $(\mathrm{p}<.029)$ y (falta de) perseverancia $(\mathrm{p}<.048)$. Así mismo, hay una mejoría significativa en la calidad de vida (Q-LES-Q) de nuestros pacientes en distintas áreas. En conclusión, el estudio pone de manifiesto que la intervención psicológica basada en Entrevista Motivacional y Terapia Cognitivoconductual permite una mejora significativa del Juego Patológico que repercute no sólo en la conducta de juego sino que también implica otros aspectos como la impulsividad y la calidad de vida.

Palabras clave: Juego patológico; Tratamiento; Entrevista Motivacional; CBT; Impulsividad; Calidad de vida.

\section{Abstract}

The aim of the current study is to determine the effectiveness of an individual psychological intervention based on the motivational interview and cognitive-behavioral therapy for the treatment of pathological gambling. A sample of 18 participants, diagnosed of pathological gambling and without any other associated comorbidity, were assessed, attended at the publicly-funded Gambling and Behavioral Addictions Unit (Consorci Sanitari de Terrassa). Median age was 46 years $(S D=12)$. All the patients achieved abstinence and completed follow-up. The Screen for Gambling Problems (NODS), Quality of Life Enjoyment and Satisfaction (Q-LES-Q), Impulsive Behavior Scale (UPPS-P), Sheehan Disability Inventory (SDI), Beck Depression Inventory (BDI) and State-Trait Anxiety Inventory (STAI) were administered pre- and posttreatment. Results showed that patients significantly reduced the problems related to gambling behavior according to the NODS score $(p<.000)$. Regarding impulsive behavior (UPPS-P), we found significant differences in negative urgency $(p<.001)$, positive urgency $(p<.001)$, (lack of) premeditation $(p<.029)$ and (lack of) perseverance $(p<.048)$. Some relevant aspects of quality of life as assessed by the Q-LES-Q had improved. In conclusion, the study shows that psychological intervention based on the motivational interview and cognitive-behavioral therapy not only significantly reduces gambling-related behavior problems but also leads to improvements in impulsivity and quality of life

Keywords: Gambling disorder; Treatment; Motivational interview; Cognitive-behavioral therapy; Impulsivity; Quality of life. 
$\mathrm{E}$ l Juego Patológico (JP) es "una conducta de juego persistente y desadaptativa que genera un malestar clínicamente significativo", según el DSM-5, Manual Diagnóstico y Estadístico de los Trastornos Mentales, Quinta Edición (APA, 2013). Dada la evidencia actual, en el DSM-5 el JP se clasifica dentro del capítulo de Trastornos relacionados con sustancias y trastornos adictivos, si bien hasta la anterior versión del DSM (APA, 2000) se situaba en el capítulo de Trastornos del control de los impulsos (Fauth-Bühler, Mann y Potenza, 2016). Tiene una prevalencia de entre 0.4-1.6\% (Grant, Odlaug y Chamberlain, 2016) y a menudo se asocia con consecuencias negativas en las diferentes áreas de la vida del paciente: un mayor porcentaje de desempleo (un $14 \%$ de pacientes con JP han perdido su empleo al menos una vez en la vida), dificultades económicas (19\%), aislamiento social, desvinculación de su red social de apoyo y repercusión en el ámbito familiar (un 54\% están divorciados) (Gerstein, Volberg y Toce, 1999; Scherrer et al., 2005). Dada la elevada prevalencia e impacto de este trastorno es importante desarrollar políticas de regulación del juego con la intención de prevenir la adicción al juego (Chóliz y SáizRuiz, 2016a; Chóliz y Sáiz-Ruiz, 2016b).

El constructo impulsividad elevada se ha descrito como un indicador de vulnerabilidad tanto para el origen como para el mantenimiento del JP (Hodgins et al., 2015). Además, la impulsividad juega un rol importante en relación a la respuesta al tratamiento, así como en la probabilidad de abandono del mismo (Ramos-Grille et al., 2013, 2015; Álvarez-Moya et al., 2011).

En relación al tratamiento, se dispone de suficiente evidencia sobre la eficacia de los tratamientos cognitivoconductuales (TCC) en el JP (Toneatto y Ladoceur, 2003), con una tendencia creciente, en cuanto al cuerpo de estudios, que avalan el uso de intervenciones basadas en Entrevista Motivacional (EM) (Cowlishaw et al., 2012; Thomas et al., 2015; Yakovenko, Quigley, Hemmelgarn, Hodgins y Ronksley, 2015). La mayoría de estudios publicados se han centrado en identificar cambios únicamente en la conducta de juego. Existen pocos estudios que analicen el efecto que el tratamiento para el Juego Patológico tiene sobre otros aspectos, como la impulsividad y la calidad de vida de los pacientes, tampoco se dispone de estudios que analicen la eficacia de la intervención psicológica combinando TCC y EM. El propósito del presente estudio es investigar la eficacia de una intervención combinada basada en EM y TCC en el tratamiento de la conducta de juego, y evaluar la eficacia de la intervención psicológica sobre la impulsividad y la calidad de vida de los pacientes.

\section{Material y métodos}

\section{Diseño}

Se trata de un estudio cuasi experimental pre y post tratamiento que incluye pacientes atendidos en la Unidad de
Juego Patológico y Adicciones comportamentales del Consorci Sanitari de Terrassa (CST) durante el año 2014. El estudio se realizó acorde a la última versión de la Declaración de Helsinki (WMA, 2013). El Comité de ética e investigación (CEIC) del CST aprobó la realización del estudio y se obtuvo el consentimiendo informado de todos los participantes.

\section{Participantes}

La muestra total está compuesta por 18 participantes mayores de 18 años, diagnosticados de JP, que hubieran alcanzado el objetivo terapéutico de la abstinencia al juego y, por lo tanto, finalizado el tratamiento. Se consideraron criterios de exclusión los pacientes con otro tipo de adicción comportamental $(n=6)$ (compras compulsivas, adicción a internet o adicción al sexo), pacientes que se mantenían en tratamiento por no haber alcanzado la abstinencia al juego $(n=21)$, y aquellos pacientes que abandonaron el tratamiento o el seguimiento $(n=5)$.

\section{Procedimiento}

Todos los participantes realizaron una primera sesión, con formato de entrevista semiestructurada, en la que se recogieron datos sociodemográficos (sexo, edad, nivel educativo, estado civil y situación laboral), información clínica relevante (comorbilidad con otro trastorno mental, uso/abuso de sustancias ilegales), así como información acerca de la conducta de juego (edad de inicio, duración del problema, frecuencia de juego, tiempo destinado al juego, cantidad de dinero gastado en el juego por semana) y síntomas de JP según criterios DSM-IV-TR (APA, 2000). Todos los participantes, con diagnóstico confirmado de $\mathrm{JP}$, fueron incluidos en un programa de tratamiento individual que combina la EM con un abordaje cognitivo-conductual (TCC).

El programa de tratamiento está protocolizado, y la mayoría de técnicas empleadas se basan en la EM, el control de estímulos, la reestructuración cognitiva (comprensión del concepto de azar, así como detectar y modificar falsas creencias acerca del juego) y la prevención de recaídas. El tratamiento se divide en dos fases: una primera fase dirigida a conseguir la abstinencia de juego, donde se utilizan técnicas de EM orientadas a potenciar tanto el discurso de cambio, como el sentimiento de autoeficacia del paciente hacia el inicio del cambio de conducta. La segunda fase del tratamiento va dirigida a mantener la abstinencia de juego, mediante técnicas de TCC como el control de estímulos, la reestucturación cognitiva y la prevención de recaídas. El tratamiento tiene una duración de 6 meses con sesiones individules semanales o quincenales de 40 minutos. Después del tratamiento, los pacientes inician un periodo de seguimiento de 6 meses, con visitas mensuales. Si el paciente no alcanza el objetivo terapéutico de la abstinencia al juego, este periodo se mantiene hasta acanzarlo. Por otro lado, si se alcanza el objetivo terapéutico, posteriormente se inicia 
un periodo de seguimiento más a largo plazo, que consiste en tres únicas visitas a los 3, 9 y 21 meses. La evaluación se llevó a cabo en dos momentos: previamente al inicio del tratamiento (pre-tratamiento) y después de la primera fase de 6 meses de seguimiento (post-tratamiento).

\section{Instrumentos}

Con el propósito de evaluar los cambios en la conducta de juego se evaluó la frecuencia y cantidad de dinero invertido en el juego; a la vez que se administró la versión en castellano del Screen for Gambling Problems (NODS) del National Opinion Research Center (NORC) DSM-IV (Becoña, 2004; Gerstein et al., 1999). Un cuestionario basado en los criterios DSM-IV, formado por 17 ítems. Las puntuaciones obtenidas están entre 0 y 10 , y permite establecer el grado de severidad: no conducta de juego (NODS 0), Juego de riesgo (NODS 1-2); Juego problema (NODS 3-4) y Juego Patológico (NODS $\geq 5$ ). La fiabilidad test-retest del instrumento es de 0.98 .

Los cambios en la calidad de vida y satisfacción se evaluaron mediante el Quality of Life Enjoyment and Satisfaction (Q-LES-Q; Endicott, Nee, Harrison y Blumenthal, 1993), una medida autoinformada de 93 ítems, divididos en 8 dominios: salud física, estado de ánimo, trabajo, actividades en casa, tiempo libre, relaciones sociales y actividad general la última semana. Dos ítems miden la satisfacció con la medicación y la satisfacción con la vida de forma global. Se responde con una escala tipo Likert de 5 puntos. Elevadas puntuaciones indican mayor satisfacción. Una elevada consistencia interna y buena validez ha sido descrita para este instrumento (Endicott et al., 1993; Ritsner, Kurs, Kostizky, Ponizovsky y Modai, 2002).

La impulsividad fue evaluada mediante la UPPS-P Impulsive Behavior Scale (Whiteside y Lynam, 2001; Cyders et al., 2007), un instrumento de 59 ítems que evalua la impulsividad como rasgo de personalidad. Incluye 5 subescalas: (falta de) premeditación, entendida com la tendencia a actuar sin pensar en possibles consecuencias $(\alpha=0.85)$; urgencia negativa, tendencia a actuar en respuesta a experiencias afectivas negativas $(\alpha=0.87)$; urgencia positiva, tendencia a actuar en respuesta a experiencias afectivas positivas $(\alpha=$ 0.93); (falta de) perseverancia, dificultad para mantenerse en una tarea $(\alpha=0.85)$ y búsqueda de sensaciones $(\alpha=$ 0.86) (MacKillop et al., 2016).

Se administró el Sheehan Disability Inventory (SDI; Sheehan, Hamett-Sheehan y Raj, 1996) con el fin de evaluar el grado de disfunción percibido. El SDI es un cuestionario autoadministrado que consta de 5 ítems que evalúan de forma subjetiva el grado de discapacidad del paciente en tres áreas básicas (laboral, social/ocio y familiar/responsabilidades del hogar), así como el grado de preocupación o estrés percibido en la última semana y el apoyo social percibido. Los cuatro primeros ítems se puntuan en una escala analógica visual numérica del 1 al $10(0=$ no discapacidad; $10=$ máxima discapacidad) y en el caso del último ítem se usan porcentajes $(0 \%=$ apoyo inexistente; $100 \%=$ apoyo ideal $)$.

Se administró también el Beck Depression Inventory (BDI; Beck, Steer y Brown, 2011) y el State-trait Anxiety Inventory (STAI; Spielberger, Gorsuch y Lushene, 1982) para evaluar la presencia de sintomatología depresiva y ansiosa, respectivamente. Las propiedades psicométricas de ambos instrumentos han sido ampliamente validadas.

Tabla 1. Principales variables sociodemográficas y de la conducta de juego

\begin{tabular}{|c|c|}
\hline Variables sociodemográficas & Media (DS) o n (\%) \\
\hline Edad & $45.8(12)$ \\
\hline Sexo (hombre) & $18(100 \%)$ \\
\hline \multicolumn{2}{|l|}{ Estado civil } \\
\hline Casado & $12(66,7 \%)$ \\
\hline Soltero & $3(16,7 \%)$ \\
\hline Divorciado & $3(16,7 \%)$ \\
\hline \multicolumn{2}{|l|}{ Nivel educativo } \\
\hline Primarios & $3(16,7 \%)$ \\
\hline Medios & $8(44,4 \%)$ \\
\hline Secundarios & $3(16,7 \%)$ \\
\hline Superiores & $3(16,7 \%)$ \\
\hline \multicolumn{2}{|l|}{ Estado laboral } \\
\hline Activo & $10(55,6 \%)$ \\
\hline En paro & $5(27,8 \%)$ \\
\hline Jubilado & $2(11,1 \%)$ \\
\hline Invalidez & $1(5,6 \%)$ \\
\hline \multicolumn{2}{|l|}{ Uso de sustancias } \\
\hline \multicolumn{2}{|l|}{ Tabaco } \\
\hline No & $16,7 \%$ \\
\hline Exfumador & $38,9 \%$ \\
\hline $\mathrm{Si}$ & $44,4 \%$ \\
\hline \multicolumn{2}{|l|}{ Alcohol } \\
\hline No & $33,3 \%$ \\
\hline Exconsumo & $5,6 \%$ \\
\hline Consumo esporádico & $50 \%$ \\
\hline Consumo regular & $11,1 \%$ \\
\hline \multicolumn{2}{|l|}{ Cannabis } \\
\hline No & $94,4 \%$ \\
\hline Exconsumo & $5,6 \%$ \\
\hline \multicolumn{2}{|l|}{ Cocaína } \\
\hline No & $94,4 \%$ \\
\hline Exconsumo & $5,6 \%$ \\
\hline
\end{tabular}

Variables de la conducta de juego

Media (DS) o n (\%)

Edad media inicio juego

$27.28(11.42)$

Tipo de juego

Máquinas tragamonedas $\quad 16(88,9 \%)$ Apuestas online $2(11,1 \%)$

Años desde inicio JP

$<1$ año

2-5 años

6-10 años

11-15 años

Recaída

$3(16,7 \%)$

$1(5,6 \%)$

Frecuencia de juego

Diario

$11(61,1 \%)$

2-4 veces/semana

1 vez a la semana

Dinero gastado/semana

$20-60 €$

$60-100 €$

100-500€ 
Tabla 2. Diferencias pre y post tratamiento en variables clínicas, relativas al juego, a la calidad de vida y a la impulsividad.

\begin{tabular}{|c|c|c|c|}
\hline Instrumento & $\begin{array}{r}\text { Pre } \\
\text { Media (DS) o n (\%) }\end{array}$ & $\begin{array}{r}\text { Post } \\
\text { Media (DS) o n (\%) }\end{array}$ & $\begin{array}{r}\text { Signifiancia } \\
\text { Valor-p }\end{array}$ \\
\hline NODS & $6.72(2.516)$ & $1.33(2.42)$ & .000 \\
\hline Discapacidad & $16.39(5.782)$ & $14.22(9)$ & .737 \\
\hline Estrés percibido & $3.59(3.242)$ & $2.39(2.725)$ & .105 \\
\hline Soporte Social percibido & $75.56(28.743)$ & $77.78(27.344)$ & .776 \\
\hline Salud física & $44.78(9)$ & $47.50(10.557)$ & .05 \\
\hline Estado de ánimo & $49.39(10.793)$ & $54.83(11.336)$ & .076 \\
\hline Trabajo & $52.40(9.095)$ & $54.92(10.942)$ & .213 \\
\hline Actividades en casa & $35(5.863)$ & $41.50(6.981)$ & .006 \\
\hline Ocio & $20.39(5.078)$ & $21.94(5.230)$ & .324 \\
\hline Relaciones sociales & $38.61(6.626)$ & $43.33(8.513)$ & .031 \\
\hline Actividad general & $46.61(9.243)$ & $54.78(9.997)$ & .078 \\
\hline Satisfacción con la medicación & $1.72(2.052)$ & $1(1.715)$ & .120 \\
\hline \multicolumn{4}{|l|}{ UPPS-P } \\
\hline Urgencia Negativa & $2.59(.373)$ & $2.10(.504)$ & .001 \\
\hline Urgencia Positiva & $2.18(.580)$ & $1.77(.509)$ & .001 \\
\hline (Falta de) Premeditación & $2.17(.423)$ & $2.01(.462)$ & .029 \\
\hline (Falta de) Perseverancia & $2.02(.415)$ & $1.82(.519)$ & .048 \\
\hline Búsqueda de Sensaciones & $1.77(.676)$ & $1.66(.494)$ & .288 \\
\hline BDI & $15.11(11.842)$ & $6.17(6.109)$ & .002 \\
\hline No depresión & $50 \%$ & $77.8 \%$ & \\
\hline Depresión Leve & $11.1 \%$ & $16.7 \%$ & \\
\hline Depresión Moderada & $27.8 \%$ & $5.6 \%$ & \\
\hline Depresión Severa & $11.1 \%$ & - & \\
\hline \multicolumn{4}{|l|}{ STAI (Pc) } \\
\hline Estado & 70.27 & 44.66 & .000 \\
\hline Rasgo & 70 & 48.11 & .001 \\
\hline
\end{tabular}

Nota. DS = Desviación estándard; Pc= Percentil; NODS = National Opinion Research Center (NORC) DSM-IV Screen for Gambling Problems (NODS); SDI = Sheehan Disability Inventory; Q-LES-Q = Quality of Life Enjoyment and Satisfaction; UPPS-P = UPPS-P Impulsive Behavior Scale; BDI = Beck Depression Inventory, STAI = The State-Trait Anxiety Inventory.

\section{Análisis estadístico}

El análisis estadístico se realizó utilizando el Paquete Estadístico para las Ciencias Sociales (SPSS, versión 21.0 para Windows). En primer lugar, se estimaron las prevalencias de las principales características sociodemográficas y clínicas. El análisis de los datos previos y posteriores a la intervención se evaluaron mediante la prueba no paramétrica de Wilcoxon para muestras relacionadas.

\section{Resultados}

Los 18 participantes eran hombres con una edad media de 45.8 años. La mayoría casados $(66.7 \%)$, con estudios medios $(44.4 \%)$ y activos laboralmente en el momento de la primera visita $(55.6 \%)$. La edad media de inicio de los problemas con el juego fue de 27.28 años, con una duración del problema de unos 2-5 años, siendo las máquinas tragamonedas la principal actividad entorno al juego, y en el momento de la primera visita no presentaban diagnósticos psiquiátricos comórbidos (Tabla 1).

Los resultados indican que después de 6 meses de tratamiento basado en EM y TCC, y de 6 meses de seguimiento los problemas asociados a la conducta de juego se habían reducido significativamente, siendo la diferencia entre la evaluación pre y post estadísticamente significativa $(\mathrm{p}<.000)$ (NODS-pre: 6.72; NODS-post: 1.33). Además, se encontraron diferencias significativas pre-post en la clínica afectiva (BDI p<.002) y ansiosa (A-Estado p<.000; A-Rasgo $\mathrm{p}<.001)$.

En relación a la impulsividad, se encontraron diferencias significativas pre-post tratamiento en las variables: urgencia negativa $(\mathrm{p}<.001)$, urgencia positiva $(\mathrm{p}<.001)$, (falta de) premeditación $(\mathrm{p}<.029)$ y (falta de) perseverancia $(p<.048)$. No se encontraron diferencias en la variable búsqueda de sensaciones.

Finalmente, los resultados sugieren que el tratamiento basado en TCC y EM también supone mejora en otros aspectos relevantes como la calidad de vida en relación a la salud física $(\mathrm{p}<.05)$, las actividades en casa $(\mathrm{p}<.006)$, las relaciones sociales $(\mathrm{p}<.031)$ y la satisfacción global $(\mathrm{p}<.014)$ evaluadas mediante el Q-LES-Q. No se encontraron diferencias estadísticamente significativas al evaluar el constructo de calidad de vida con el instrumento SDI.

La Tabla 2 muestra las diferencias pre y post tratamiento de las variables clínicas, las relacionadas con la conducta de juego, y las variables de calidad de vida e impulsividad. 


\section{Discusión}

Los resultados de nuestro estudio ponen de manifiesto que la intervención psicológica basada en EM y TCC permite mejoras significativas, no sólo de la conducta de juego, que es el objetivo principal; sino que también supone mejoras en otros aspectos como la conducta impulsiva y la calidad de vida de los pacientes.

Las mejoras en relación a la conducta de juego, no sólo hacen referencia a la puntuación total de la escala NODS, sino también a otras variables asociadas a la conducta de juego como son la frecuencia y la inversión semanal realizada por los pacientes. Estos resultados coinciden con los sugeridos en dos meta-análisis recientes, donde queda ampliamente demostrada la eficacia de la intervención basada en EM y TCC para conseguir cambios y mejoras en la conducta de juego (Cowlishaw et al., 2012 y Yakovenko et al., 2015).

Los resultados de nuestro estudio también permiten concluir que el tratamiento psicológico realizado proporciona mejoras significativas en la valoración general de la calidad de vida del paciente, concretamente en áreas como la salud física, las actividades en casa y las relaciones sociales. Con la resolución del problema de juego, los pacientes refieren mejoras en ámbitos de la vida que se encuentran alterados mientras la conducta de juego está activa, sea debido a la propia conducta de juego o a las consecuencias ocasionadas por el juego. Estos resultados corroboran los resultados de los estudios de Carlbring, Degerman, Jonsson y Andersson (2012) y de Pasche (2013). Si bien disponemos de evidencias sobre el efecto positivo de la intervención psicológica en la mejora de la calidad de vida de estos pacientes, faltan estudios que permitan identificar qué intervenciones son más eficaces y cual es la relación que se establece entre las mejoras en la conducta de juego y las mejoras en la calidad de vida.

En relación a la impulsividad, a partir del modelo multifactorial de Whiteside y Lynam (2001), y posteriormente Cyders y Smith (2008), se propone que los diferentes aspectos de este modelo (urgencia positiva, urgencia negativa, (falta de) premeditación, (falta de) perseverancia y búsqueda de sensaciones) se comportan como factores de riesgo para conductas disfuncionales y desadaptativas (Verdejo-Garcia, Bechara, Recknor y Pérez-García, 2007). En muestras de JP, Michalczuk, Bowden-Jones, Verdejo-García y Clark (2011) describen que los jugadores obtienen puntuaciones elevadas en todas las subescalas; si bien, el tamaño del efecto es mayor en las escalas de urgencia positiva y negativa. Disponemos de pocos estudios con muestras de jugadores que analicen cambios y mejoras en la impulsividad una vez finalizado el tratamiento. Acorde a esto, los resultados de nuestro estudio permiten identificar mejoras en el funcionamiento de estos pacientes, sobretodo en relación a la urgencia positiva y negativa. Esto podría sugerir que la intervención centrada en la identificación y afrontamiento de situaciones de riesgo, de problemas y/o emo- ciones, contribuye a una mayor autoeficacia del paciente, percibiéndose con más capacidad para afrontar estas situaciones, y haciéndolo de forma más adaptativa.

Nuestro estudio tiene algunas fortalezas y limitaciones que deben ser comentadas. La principal fortaleza es haber abierto el foco de estudio, no sólo centrándonos en la conducta de juego tras el tratamiento, sino también en aspectos como la impulsividad y la calidad de vida. Por otro lado, algunas de las limitaciones son el tamaño de la muestra y la falta de un grupo control lo cual impide identificar el tamaño del efecto y la generalización de los resultados obtenidos. En el momento actual se está trabajando para aumentar el tamaño muestral, así como para conocer cuales son las características de aquellos pacientes que se mantienen en el seguimiento lo que permitirá consolidar los resultados y conocer cuales deben ser las características de los tratamientos para favorecer la adherencia

En conclusion, nuestros resultados corroboran la eficacia de la TCC, en combinación con la EM para reducir la conducta de juego y evidencian de forma original su efecto sobre la impulsividad y la calidad de vida de los pacientes con JP.

\section{Conflicto de intereses}

Los autores declaran no presentar conflictos de intereses.

\section{Referencias}

Álvarez-Moya, E. M., Ochoa, C., Jiménez-Murcia, S., Aymami, M. N., Gómez-Peña, M., Fernández-Aranda, F., ... Menchón, J. M. (2011). Effect of executive functioning, decision-making and self-reported impulsivity on the treatment outcome of pathological gambling. Journal of Psychiatry EO Neuroscience, 36, 165-175. doi:10.1503/jpn.090095.

American Psychiatric Association (2000). Diagnostic and Statistical Manual of Mental Disorders (4th ed. text revision). Washington, DC: American Psychiatric Association.

American Psychiatric Association (2013). Diagnostic and Statistical Manual of Mental Disorders (5th edition). Washington, DC: American Psychiatric Association.

Beck, A. T., Steer, R. A. y Brown, G. K. (2011). Manual. BDI-II. Inventario de Depresión de Beck-II (Adaptación española: Sanz, J. y Vázquez, C.). Madrid: Pearson.

Becoña, E. (2004). Prevalencia del juego patológico en Galicia mediante el NODS ¿Descenso de la prevalencia o mejor evaluación del trastorno? Adicciones, 16, 173-184. doi: 10.20882/adicciones.399

Carlbring, P., Degerman, N., Jonsson, J. y Andersson, G. (2012). Internet-based treatment of pathological gambling with a three-year follow-up. Cognitive Behaviour Therapy, 41, 321-334. doi:10.1080/16506073.2012.689323.

Chóliz, M. y Sáiz-Ruiz, J. (2016a). Regular el juego para prevenir la adicción: hoy más necesario que nunca. Adicciones, 28, 174-181. doi:10.20882/adicciones.820. 
Chóliz, M. y Sáiz-Ruiz, J. (2016b). ¿Por qué (también) es tan difícil legislar sobre el juego en España? Un "dejà vu" de lo ocurrido con el alcohol. Adicciones, 28, 189-193. doi:10.20882/adicciones.886.

Cowlishaw, S., Merkouris, S., Dowling, N., Anderson, C., Jackson, A. y Thomas, S. (2012). Psychological therapies for pathological and problem gambling. Cochrane Database of Systematic Reviews (Online), 1, 1-91. doi:10.1002/14651858. CD008937.pub2.

Cyders, M. A., Smith, G. T., Spillane, N. S., Fischer, S., Annus, A. M. y Peterson, C. (2007). Integration of impulsivity and positive mood to predict risky behavior: development and validation of a measure of positive urgency. Psychological Assessment, 19, 107-118. doi: 10.1037/10403590.19.1.107.

Cyders, M. A. y Smith, G. T. (2008). Clarifying the role of personality dispositions in risk for increased gambling behavior. Personality and Individual Differences, 45, 503-508.

Endicott, J., Nee, J., Harrison, W. y Blumenthal, R. (1993). Quality of Life Enjoyment and Satisfaction Questionnaire: A new measure. Psychopharmacology Bulletin, 29, 321-326.

Fauth-Bühler, M., Mann, K. y Potenza, M. N. (2016). Pathological gambling: A review of the neurobiological evidence relevant for its classification as an addictive disorder. Addiction Biology, 3, 1-13. doi:10.1111/adb.12378.

Gerstein, D. R., Volberg, R. A., Toce, M. T., Harwood, H., Johnson, R., Buie, T., ...Sinclair, S. (1999). Gambling impact and behavior study. Report to the National Gambling Impact Study Commission. Chicago, in NORC at the University of Chicago.

Grant, J. E., Odlaug, B. L. y Chamberlain, S. R. (2016) Neural and psychological underpinning of gambling disorder: A review. Progress in Neuro-psychopharmacoly and Biological Psychiatry, 65, 188-193. doi:10.1016/j.pnpbp.2015.10.007.

Hodgins, D. C. y Holub, A. (2015). Components of Impulsivity in Gambling Disorder. International Journal of Mental Health and Addiction, 13, 699-711. doi:10.1007/s11469015-9572-z.

MacKillop, J., Weafer, J., Gray, J., Oshri, A., Palmer, A. y de Wit, H. (2016). The latent structure of impulsivity: impulsive choice, impulsive action, and impulsive personality traits. Psychopharmacology, 233, 3361-3370. doi:10.1007/ s00213-016-4372-0.

Michalczuk, R., Bowden-Jones, H., Verdejo-Garcia, A. y Clark, L. (2011). Impulsivity and cognitive distortions in pathological gamblers attending the UK National Problem Gambling Clinic: a preliminary report. Psychological Medicine, 41, 2625-2635. doi: 10.1017/S003329171100095X.

Pasche, S. C., Sinclair, H., Collins, P., Pretorius, A., Grant, J. E. y Stein, D. J. (2013). The effectiveness of a cognitive-behavioral intervention for pathological gambling: A country-wide study. Annals of Clinical Psychiatry, 25, 250256.
Ramos-Grille, I., Gomà-i-Freixanet, M., Aragay, N., Valero, S. y Vallès, V. (2013). The role of personality in the prediction of treatment outcome in pathological gamblers: a follow-up study. Psychological Assessment, 25, 599-605. doi:10.1037/a0031930.

Ramos-Grille, I., Gomà-i-Freixanet, M., Aragay, N., Valero, S. y Vallès, V. (2015). Predicting treatment failure in pathological gambling: the role of personality traits. Addictive Behaviors, 43, 54-59. doi: 10.1016/j.addbeh.2014.12.010.

Ritsner, M., Kurs, R., Kostizky, H., Ponizovsky, A. y Modai, I. (2002). Subjective quality of life in severely mentally ill patients: a compatison of tow instruments. Quality of Life Research, 11, 553-561.

Scherrer, J. F., Xian, H., Shah, K. R., Volberg, R., Slutske, W. y Eisen, S. A. (2005). Effect of genes, environment, and lifetime co-occurring disorders on health-related quality of life in problem and pathological gamblers. Archives of General Psychiatry, 62, 677-683. doi:10.1001/archpsyc.62.6.677.

Sheehan, D. V., Hamett-Sheehan, K, y Raj, B. A. (1996). The measurement of disability. International Clinical Psychopharmacology, 11, 89-95.

Spielberger, C. D., Gorsuch, R. L. y Lushene, R. (1982). Manual del Cuestionario de Ansiedad Estado/Rasgo (STAI). Madrid, España: TEA Ediciones

Thomas, S. A., Merkouris, S. S., Browning, C. J., Radermacher, H., Feldman, S., Enticott, J. y Jackson, A. C. (2015). The PROblem Gambling RESearch Study (PROGRESS) research protocol: a pragmatic randomised controlled trial of psychological interventions for problem gambling. BMJ Open, 5, e009385. doi:10.1136/bmjopen-2015-009385.

Toneatto, T. y Ladoceur, R. (2003). Treatment of pathological gambling: a critical reveiw of the literature. Psychology of Addictive Behaviours, 27, 284-92. doi:10.1037/0893164X.17.4.284.

Verdejo-García, A., Bechara, A., Recknor, E. C. y Pérez-García, M. (2007). Negative emotion-driven impulsivity predicts substance dependence problems. Drug and Alcohol Dependence, 91, 213-219. doi:10.1016/j.drugalcdep.2007.05.025.

Whiteside, S. P. y Lynam, D. R. (2001). The five factor model and impulsivity: using a structural model of personality to understand impulsivity. Personality and Individual Differences, 30, 669-689. doi:10.1016/S0191-8869(00)00064-7.

World Medical Association (2013). World Medical Association Declaration of Helsinki: Ethical Principles for Medical Research Involving Human Subjects. Journal of the American Medical Association, 310, 2191-2194. doi:10.1001/ jama.2013.281053.

Yakovenko, I., Quigley, L., Hemmelgarn, B. R., Hodgins, D. C. y Ronksley, P. (2015). The efficacy of motivational interviewing for disordered gambling: systematic review and meta-analysis. Addictive Behaviors, 43, 72-82. doi:10.1016/j. addbeh.2014.12.011. 